\title{
TBM
}

\section{The effect of a locally adapted, secondary stroke risk factor self-management program on medication adherence among veterans with stroke/TIA}

\author{
Teresa M. Damush, Ph.D., ${ }^{1}$ Laura Myers, Ph.D., Jane A. Anderson, Ph.D., A.N.P., ${ }^{2}$ Zhangsheng Yu, Ph.D., \\ Susan Ofner, M.S., ${ }^{1}$ Gloria Nicholas, R.N., ${ }^{1}$ Barbara Kimmel, M.S., ${ }^{1}$ Arlene A. Schmid, Ph.D., O.T.R., ${ }^{1}$ \\ Thomas Kent, M.D., ${ }^{2}$ Linda S. Williams, M.D. ${ }^{1}$
}

\begin{abstract}
VA HSRD QUERI Center and HSRD
CHIC Center,

Indiana University School of

Medicine, Regenstrief Institute, Inc, Indianapolis, IN, USA

${ }^{2}$ VA HSRD Coin Center, Michael DeBakey VAMC,

Baylor College of Medicine, Houston, TX, USA

Correspondence to: T Damush

tdamush@iupui.edu
\end{abstract}

Cite this as: TBM 2016;6:457-468 doi: 10.1007/s13142-015-0348-6

\section{Abstract}

We targeted stroke/transient ischemic attack (TIA) survivors to engage in self-management practices to manage secondary stroke risk factors. We conducted a randomized, regional pilot trial of a locally adapted, secondary stroke prevention program. We implemented the program at two Veterans Administration Medical Centers. Program sessions targeted stroke risk factor self-management. Specifically, we evaluated the effect of the program on the reach, implementation, and effectiveness on patient selfefficacy; stroke-specific, health-related quality of life; and medication adherence for the prevalent stroke risk factors: (1) diabetes, (2) hypertension, and (3) hyperlipidemia. Medication possession ratios were calculated to evaluate medication adherence using VA pharmacy benefits data pre ( 6 months prior) and post ( 6 months after) the stroke/TIA event. Based upon the literature standard of $80 \%$ compliance rate, we dichotomized compliance and modeled the data using logistical regression. Final sample included 174 veterans with an acute stroke or TIA who were randomized to receive either the intervention $(n=87)$ or attention control program $(n=87)$. Patient selfefficacy and stroke-specific, health-related quality of life at 6 months did not significantly differ between groups. We found improvements in medication adherence within the intervention group. In the intervention group, the odds of compliance with diabetes medications post-stroke were significantly larger than the odds of compliance prior to the stroke (odds ratio $=3.45(95 \% \mathrm{Cl}=1.08-10.96)$. For compliance to hypertension medications, the intervention group showed significantly greater odds of compliance post intervention than pre intervention (odds ratio $=3.68$ (95\% Cl=1.81-7.48). The control group showed no difference in compliance rates from baseline to follow-up. For adherence to hypercholesterolemia medications, both the intervention (odds ratio $=5.98(95 \% \mathrm{Cl}=2.81-12.76)$ and control groups (odds ratio $=3.83(95 \% \mathrm{Cl}=1.83-$ 8.01), had significant increases in the odds of compliance to statin medications; however, the comparison of changes in log odds of compliance between these two groups showed that the increases were not significantly different. We observed within group improvements in medication adherence among those receiving a post-

\section{Implications}

Policy: Nursing work tasks should be allocated for patient education to foster self-management practices during post-acute stroke care.

Research: Medication possession ratios from pharmacy data may serve as objective medication adherence outcomes.

Practice: Inclusion of medication adherence strategies during post-acute stroke care including how to refill, and strategies for adhering to prescriptions may improve patient adherence.

stroke risk factor self-management program suggesting that a self-management format may be feasible to enable adherence to prescribed medications to reduce secondary stroke risk after stroke in concordance with guideline care. Additional research is needed to enhance intervention components to improve effectiveness outcomes.

\section{Keywords}

Medication adherence, Secondary stroke prevention, Local adaptation

\section{BACKGROUND}

Stroke is the fifth leading cause of death [1], produces the greatest number of hospitalizations for neurological disease, and is the leading cause of adult acuteonset and long-term disability in the USA [2]. In addition to physical disabilities, stroke survivors may experience disruptions to their daily roles (e.g., work, family) which may affect their health-related quality of life [3-5].

Moreover, patients who have had a stroke or a transient ischemic attack (TIA) are at an increased risk for recurrent stroke and death [2]. Modifiable risk factors are most effectively managed through a combination of lifestyle and medication management and thus require the combined and collaborative efforts of 
providers and patients. Although many stroke risk factors are modifiable, often, patients with stroke or TIA do not have adequate control of their stroke risk factors [6]. Despite knowledge of the methods and impact of risk reduction, providers may not systematically counsel or treat patients with behavioral or medical interventions specifically for stroke prevention [7].

Currently, we are aware of no systematic programs or support available in the Veterans Health Administration (VHA) to improve guideline adherence for secondary stroke risk factor management. Although some programs exist to support specific primary risk factor management (e.g., diabetes or hypertension), these are not routinely targeted to veterans with recent stroke or TIA. Further, there is less support for management of behavioral risk factors and for improving stroke survivors' education and self-management practices. Yet, educating stroke survivors on stroke risk management is mandated by the VA/DoD stroke rehabilitation guidelines [8,9], championed by the American Stroke Association Get with the Guidelines (GWTG) Stroke [10, 11], and included in the Joint Commission criteria for stroke care [12, 13].

We utilized intervention mapping [14] to systematically adapt existing stroke prevention tools at two Veterans Administration Medical Centers (VAMCs). In that process, we conducted a formative evaluation and queried both stroke clinical providers and stroke/ TIA survivors on barriers and facilitators to systematic secondary stroke prevention delivery in VHA, and received feedback by key stakeholders on how to best modify existing stroke prevention tools to veterans and the VHA system of healthcare [7]. Based on the intervention mapping results, we developed a Veterans Stroke Prevention Program (VSPP) and locally adapted the implementation of VSPP at two VHA facilities. Like the Stroke PROTECT program, a provider- and patient-focused program to implement evidence-based, stroke prevention strategies at the time of discharge from acute stroke care [15], and in concert with the Chronic Care Model [16], we targeted both providers and patients for medication adherence and lifestyle modification to promote patient selfmanagement and reduce stroke risk factors for secondary stroke prevention. Based on the American Stroke Association and the VHA/Department of Defense guidelines and feedback by both stroke survivors and clinical providers, we have chosen to implement this program at the time of hospitalization for stroke or transient ischemic attack (TIA) given that changes made around hospitalization may be more likely to be sustained. In addition, our preliminary data from stroke survivors suggest that stroke survivors are ready to make lifestyle changes shortly after their stroke hospitalization $[17,18]$. The specific aim of this study was to conduct a randomized controlled pilot trial of the locally implementation of VSPP at two VAMCs to evaluate the impact of the program on patient selfefficacy, stroke self-management practices of medication adherence, and stroke-specific, health-related quality of life (SSQoL) at 6 months post-stroke.

\section{METHODS}

Design-We conducted a pragmatic, randomized controlled pilot study to implement a tailored, patientfocused VA stroke prevention program (VSPP). The intervention was based upon the current guideline practice recommendations and the input from the formative evaluation for enhancement of secondary stroke prevention practices [7]. The local institutional review boards at both sites approved all research reported in this paper and participants provided written consent. The pragmatic design allowed a degree of flexibility in how the intervention was implemented, no incentives were offered for measurement follow-up, and we utilized intention to treat analyses [19].

Setting-The research study occurred at two Veterans Administration Medical Centers (VAMCs). Both sites provide acute stroke services and are designated as VA Primary Stroke Centers, offering acute stroke care services including time sensitive thrombolysis $24 \mathrm{~h}$ a day across 7 days per week.

Subject identification-We prospectively recruited patients hospitalized for stroke at the two VAMCs. Subjects were identified during their stroke or TIA admission and emergency room visits. For all potential study subjects, permission to discuss the study with the patient was obtained from the attending physician prior to recruitment. Study personnel at each site conducted daily screenings of neurology, emergency service, medicine, and rehabilitation admissions (including review of electronic medical record and/or written admission logs and verbal contact with providers at both sites) to identify all patients with acute ischemic stroke/TIA. Patients who did not wish to participate in this study were excluded.

Patient eligibility-The criteria included the following: (1) age 18 or older, (2) acute diagnosis of ischemic stroke or TIA within the past 12 months, (3) able to speak and understand English, (4) no severe cognitive impairment, (5) access to a telephone, (6) willing to follow-up in VA outpatient care, (7) had a previous outpatient primary care visit during the past 12 months in VA outpatient care, (8) willing to attend all individual phone and group meetings during the 6 -month intervention, and (9) life expectancy of at least 6 months as defined by the patient's neurologist at stroke event. Cognitive screening was conducted using the Short Portable Mental Status Questionnaire [21], which assesses short- and long-term memory and orientation. This screener has been validated in a study of older community-dwelling adults [22] and we have also successfully used this screener in our VA study of post-stroke depression [5, 20]. All patients with a score $>6$ were enrolled, as this score has previously identified patients without severe dementia, which would decrease reliability of selfreported symptoms and satisfaction, while allowing the inclusion of patients with cognitive effects of stroke. Language screening was conducted using the National Institutes of Health Stroke Scale, commands and language questions [9, 23]. Patients with significant language comprehension (commands score $>0$ ) or receptive language deficits (aphasia score $\geq 2$ ) were excluded. 
Consent and enrollment-We obtained written consent, conducted the baseline assessment, and enrolled participants into the randomized controlled trial. Randomization to one of two groups (self-management or usual care attention control) was completed using computer-generated assignment, was stratified by stroke vs. TIA diagnosis; and within the subjects with stroke, randomized by receipt of inpatient rehabilitation to control for stroke severity. Enrollment, written informed consent, and other patient variables were collected at baseline (prior to hospital discharge or within 12 weeks of discharge). Patients assigned to attention control received usual care and stroke education pamphlets and general phone calls to assess stroke symptoms (with no self-management training) at identical time points as intervention patients as an active attention control.

\section{Intervention}

Patient-based VSPP intervention-The two sites locally tailored the VSPP patient intervention. The stroke selfmanagement program applied theoretical concepts of Bandura's self-efficacy [24] to the intervention components: verbal persuasions, social modeling/vicarious experiences, past achievements, and reinterpretation of sensations/physical State. Participants received up to 6 biweekly telephone sessions to deliver the stroke selfmanagement program or a general telephone call program that mimicked the intervention schedule. Sessions targeted patient stroke self-management and self-efficacy. We based our self-management intervention on the Stanford Chronic Disease Self-Management Program [25, 26], a program centered on enhancing patient selfefficacy to manage symptoms. The topics included expectations after stroke, negative/positive thinking, addressing fears, meaningful activities, follow-up medical visits, communication with providers and caregivers, adapting/coping with disabilities, medication adherence, physical activity to improve mood and energy, access to community resources, and stroke risk factor modification. Each session targeted building self-efficacy to perform specified self-management behaviors using goal setting and behavioral contracting. Each patient was coached to choose at least one specific goal to work on in each session, including the specifics of when, where, and for how long they would do the specific behavior. Patients rated their confidence in carrying out each behavior and were encouraged to only select behaviors that they rated a 7 out of a possible 10 (completely confident in doing). In the follow-up telephone calls, patients received individualized feedback about their progress toward their selected goal(s) and were encouraged to continue to work on the chosen behavior or to select or add a new behavior goal for the next 2 weeks.

\section{Measured outcomes}

To convey the degree of implementation of the VSPP, we applied the RE-AIM framework to guide the reporting of our outcomes on reach, implementation, and effectiveness [27]. We defined reach as the proportion of the eligible population enrolled into our program at the two sites. Implementation is the extent that our intervention was delivered as intended. We define effectiveness as the impact of the implemented VSPP on patient outcomes of self-efficacy, medication adherence, and stroke-specific, health-related quality of life.

Self-efficacy (i.e., confidence to perform specific behaviors) was measured using established scales designed to measure a patient's confidence level to manage stroke self symptoms, communicate with their providers, and engage in self-management behaviors (e.g., exercise regularly) [28]. We have used these scales in our previous research where patient's rate their self-efficacy on a 1-10 scale where 10 denotes high self-efficacy.

Medication adherence-Given that guideline treatment for stroke and TIA secondary prevention calls for medication therapy for strong risk factors of comorbid diabetes, hypertension, and hyperlipidemia, medication adherence was promoted in the intervention as a self-management strategy [29]. We evaluated medication adherence using a similar method as previously reported utilizing medication possession ratios [30, 31]. We identified study participants discharged on medications for treatment of these conditions as follows (each patient could be treated for multiple conditions):

a. Diabetes ( $n=46$ participants): The diabetes cohort included all subjects with medical records indicating at least one oral anti-diabetic drug was prescribed prior to admissions or at discharge or those on insulin plus oral medications.

b. Hyperlipidemia $(n=143)$ : The hyperlipidemia cohort included subjects with an LDL-c $\geq 100$ in the most recent LDL measured prior to the admission or during hospitalization with medical records data that indicated at least one statin was prescribed prior to admissions or at discharge.

c. Hypertension $(n=149)$ : The hypertension cohort included subjects with medical records data that indicated blood pressure at discharge was uncontrolled (defined as $>140 / 90$ for non-diabetic and $>130 / 80$ for diabetic) or was prescribed at least one anti-hypertensive at discharge or was already taking an anti-hypertensive at admission.

Subjects with a diagnosis of diabetes, hyperlipidemia, and/or hypertension were followed for 6 months (180 days) following hospital discharge, or until date of death if dead, to assess medication adherence poststroke. We used VA electronic medication data and were guided by a standard list of medications advocated in the stroke risk factor management program for the three risk factors and available through the VHA PBM (pharmacy benefits management) pharmacy formulary (see Appendix A). The PBM database contains outpatient prescriptions for all medications dispensed from a VA pharmacy. To assess medication adherence, we 
calculated medication prescription ratios (MPR) in the 6 months before the stroke/TIA hospitalization and in the 6 months post-stroke/TIA discharge. MPR is defined as a continuous measure of medication availability [32] within each therapeutic drug class for the three risk factors (see Appendix A) and is based upon any medication within the drug class. MPR was calculated as the number of days on a medication within its therapeutic class divided by the number of days in the follow-up period (i.e., 180 days). If multiple drugs were prescribed in a class, an MPR was calculated for each drug and then averaged. For each therapeutic class, we then dichotomized the average MPR defining a patient adherent if their MPR $\geq 80 \%$ based upon prior studies that defined adherence as $80 \%$ or greater [31, 33].

Stroke-specific, health-related quality of life was assessed with the SS-QOL [3, 34]. This 62-item instrument assesses 12 domains relevant to stroke patients including energy, mobility, work, upper extremity function, ADLs, family roles, social roles, vision, language, thinking, mood, and personality. Our work has shown that the SS-QOL has good psychometric properties and that it provides a more meaningful assessment of overall post-stroke HRQOL than the SF-36 [3, 34]. In addition to the separate domains, an overall score was calculated.

\section{Analyses plan}

Baseline characteristics were summarized using mean (standard deviation (SD)) or proportion by groups for continuous and categorical variables and compared using $t$ tests or chi-square tests. For each of the three types of compliance, we summarize the mean (SD) of MPR by intervention/control groups at each time point ( 6 months prior to the acute stroke/TIA versus 6 months post-acute stroke/TIA event). Good adherence was defined as having MPR $\geq 80 \%[31,33]$. The dichotomous outcome for each of the three medication adherence types was modeled using repeated measurement logistic regression with terms for site;

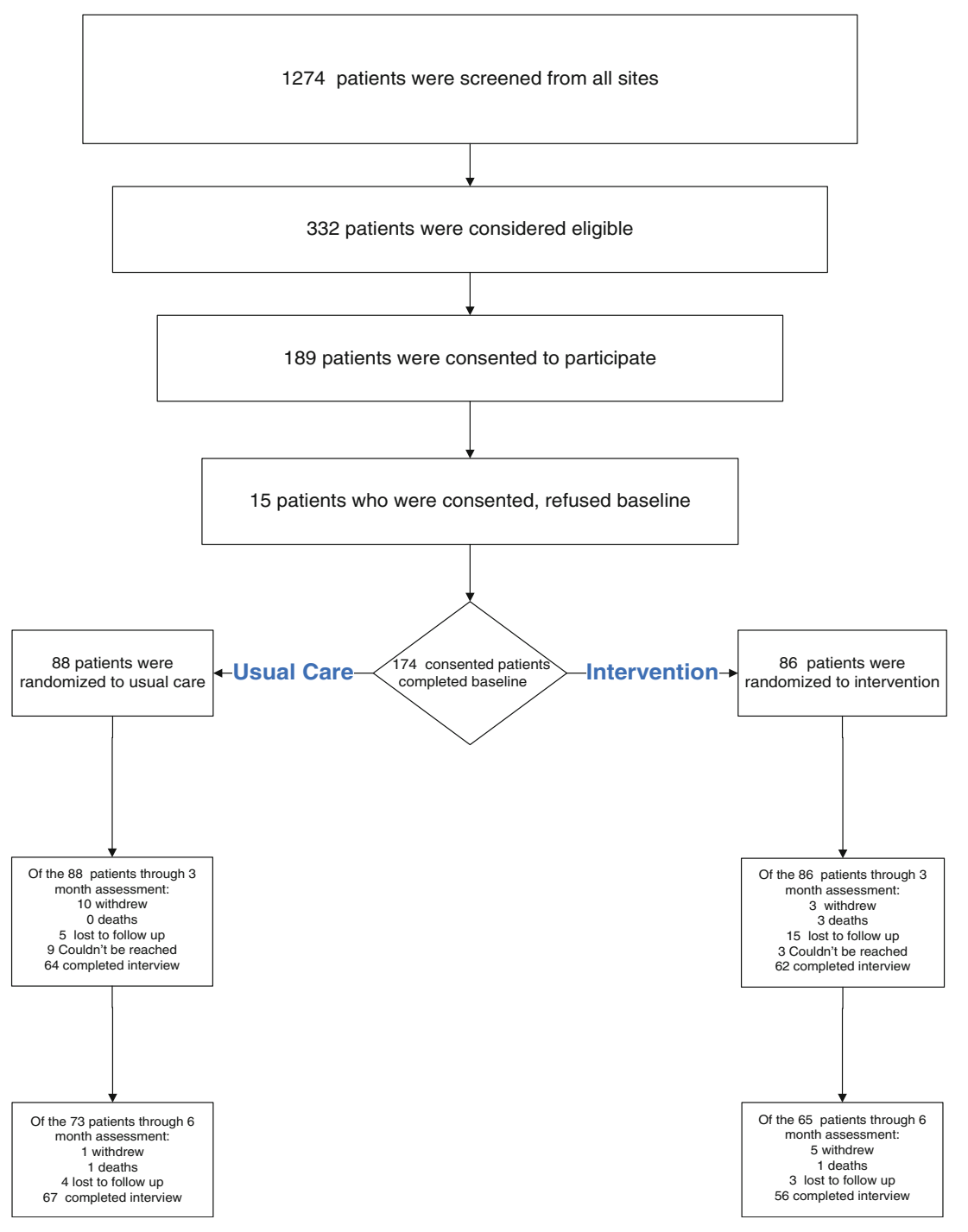


strata (stroke, stroke with inpatient rehabilitation, TIA); randomization group; time; and the group by time interaction. A random subject effect was included to incorporate the correlation of repeated measurements into the model. The model yielded estimates and $95 \%$ confidence intervals of the ratio of odds of good adherence at 6 months post event versus 6 months pre event for each group. For patient selfefficacy and SSQOL, scores at 6 months were compared between groups by using a mixed model with terms for site, strata, baseline score, group, month, and the group by month interaction. A random subject effect was used to correlate observations from the same subject. The analyses were conducted using SAS/ STAT ${ }^{\circledR}$ software, Version 9.3 of the SAS System for Windows. Copyright (C) [2010] SAS Institute Inc. SAS and all other SAS Institute Inc. product or service names are registered trademarks or trademarks of SAS Institute Inc., Cary, NC, USA.

\section{RESULTS}

Reach-In Fig. 1, we display the prospective total number of patients screened $(n=1274)$ at both medical facilities during 2009-2011. Of these, we determined $332(26 \%)$ were eligible to participate and consented $174(52.4 \%)$ to participate in the implementation study. The most frequent reason for ineligibility was a confirmed diagnosis other than stroke/TIA. The final sample included 174 veteran patients with an acute stroke/TIA who were consented, completed a baseline interview, and were randomized to receive either the Veteran Secondary Stroke Prevention program $(n=87)$ or an attention control program $(n=87)$. Table 1 demonstrated the randomized groups did not differ significantly on any of the demographic characteristics.

Implementation-As part of the pragmatic design, each site tailored the delivery of the secondary stroke self-management. An example of the intervention fidelity tracking sheet by the case manager and commonly reported patient goals are listed in Appendices B and C, respectively. Based upon the case manager fidelity tracking spreadsheets, at site 1 , the average number of stroke self-management sessions delivered to the intervention subjects by a nurse case manager either by telephone or in person at the medical center after discharge was 5 (90\% intervention subjects received). At site 2, $74 \%$ intervention subjects received the self-management intervention materials with nurse case management during the acute hospitalization and the follow-up neurology outpatient visit. At site 1, the nurse case manager provided patient support concurrent to usual outpatient care and at site 2 , the nurse case manager provided patient support during hospitalization within a stroke unit and stroke outpatient clinic. All intervention participants received all of the self-management materials;

Table 1 | Participant demographics by randomized group

\begin{tabular}{|c|c|c|c|c|}
\hline & & \multicolumn{3}{|l|}{ All } \\
\hline & & $\begin{array}{l}\text { Control } \\
n=87\end{array}$ & $\begin{array}{l}\text { Intervention } \\
n=87\end{array}$ & $p$ value* \\
\hline \multirow[t]{5}{*}{ Strata } & Stroke & $49(56.3 \%)$ & $49(56.3 \%)$ & \\
\hline & Stroke with rehabilitation & $18(20.7 \%)$ & $17(19.5 \%)$ & \\
\hline & $\overline{T I A}$ & $20(23.0 \%)$ & $21(24.1 \%)$ & \\
\hline & Age mean $\pm S D$ & $62.1 \pm 9.4$ & $60.4 \pm 9.5$ & 0.2423 \\
\hline & (median: min, max) & $(62: 32,86)$ & $(61: 30,78)$ & \\
\hline \multirow[t]{2}{*}{ Gender } & Male & $85(97.7 \%)$ & $84(96.6 \%)$ & 0.9999 \\
\hline & Female & $2(2.3 \%)$ & $3(3.4 \%)$ & \\
\hline \multirow[t]{5}{*}{ Race } & American Indian/Alaska native & $1(1.2 \%)$ & $1(1.2 \%)$ & 0.6246 \\
\hline & Native Hawaiian or other Pacific islander & $1(1.2 \%)$ & $0(0.0 \%)$ & \\
\hline & Black or African-American & $29(33.3 \%)$ & $24(27.6 \%)$ & \\
\hline & White & $51(58.6 \%)$ & $54(62.1 \%)$ & \\
\hline & More than one race & $0(0.0 \%)$ & $1(1.2 \%)$ & \\
\hline Ethnicity & Hispanic & $4(4.6 \%)$ & $11(12.6 \%)$ & 0.0924 \\
\hline \multirow[t]{6}{*}{ Education level } & Less than high school & $10(11.5 \%)$ & $7(8.1 \%)$ & 0.4912 \\
\hline & High school/GED & $23(26.4 \%)$ & $31(35.6 \%)$ & \\
\hline & Some college or trade & $33(37.9 \%)$ & $26(29.9 \%)$ & \\
\hline & College graduate & $8(9.2 \%)$ & $12(13.8 \%)$ & \\
\hline & Graduate school or more & $5(5.8 \%)$ & $4(4.6 \%)$ & \\
\hline & Missing & $8(9.2 \%)$ & $7(8.1 \%)$ & \\
\hline \multirow[t]{4}{*}{ Living status } & Alone & $25(28.7 \%)$ & $18(20.7 \%)$ & 0.2952 \\
\hline & with only 1 other person & $37(42.5 \%)$ & $46(52.9 \%)$ & \\
\hline & with $2+$ & $25(28.7 \%)$ & $21(24.1 \%)$ & \\
\hline & Missing & $0(0.0 \%)$ & $2(2.3 \%)$ & \\
\hline
\end{tabular}

$5.7 \%$ of the participants in the control group and $8.1 \%$ of the participants in the intervention group reported their race as "don't know" or this data was missing. These data were excluded from the demographic comparisons of race 
Table 2 | Patient self-reported outcomes of self-efficacy and stroke-specific quality of life (SSQoL)

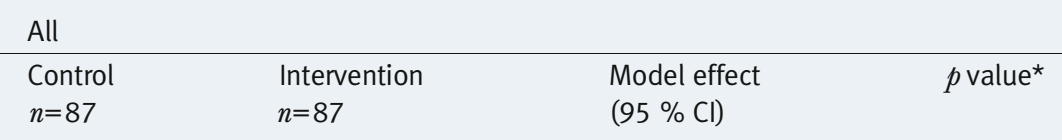

SSQoL overall score

\begin{tabular}{|c|c|c|c|c|}
\hline Baseline & $3.92(0.72)$ & $3.72(0.68)$ & $-0.04(-0.26,0.18)$ & \\
\hline 6 months & $3.94(0.82)$ & $3.82(0.88)$ & & \\
\hline Self-efficacy baseline & $8.30(1.6)$ & $7.72(1.68)$ & $0.12(-0.41,0.65)$ & 0.66 \\
\hline Self-efficacy 6 months & $8.08(1.8)$ & $7.90(1.78)$ & & \\
\hline
\end{tabular}

A higher score indicates better functioning. SSQoL scores range from 1 to 5 and self-efficacy scores range from 1 to 10 . The model adjusted for randomization group, site, strata, baseline score, month, and the group by month interaction

however, when subjects attended less sessions with a nurse case manager, they missed an opportunity to engage in self-management skill building specifically in goal setting and problem-solving.

\section{Effectiveness}

SSQoL and self-efficacy-Table 2 includes the means (standard deviations) for the patient self-reported outcomes of self-efficacy to manage stroke symptoms and health and stroke-specific health-related quality of life at baseline and 6 months. Although self-efficacy increased in the intervention group and decreased in the control group over time, the modeled changes between randomization groups on these self-efficacy and stroke-specific, health-related quality of life were nonsignificant at 6 months.

Medication adherence-Table 3 lists the proportion of participants with stroke/TIA by randomization group for each of the three comorbid, clinical cohorts (diabetes, hyperlipidemia, and hypertension) and the respective Medication Possession Ratios 180 days (6 months) prior and 6 months post-stroke/TIA event. Table 4 displays the within and between groups modeling of medication adherence at $80 \%$ or greater within the intervention group, the odds of compliance to diabetes medications post-stroke were significantly larger than the odds of compliance prior to the stroke (odds ratio $=3.45(95 \% \mathrm{CI}=1.08-10.96)$ $p<0.04)$. The control group saw a decrease in the likelihood of compliance to diabetes medications from pre- to post-stroke hospitalization (odds ratio= 0.51 (95\% CI=0.10-2.70), $p<0.42$ ); however, the decrease was not significant. The between comparison (ratio) of the odds (post versus pre) in the intervention group to the odds in the control group was not significant (odds ratio $=6.74 \quad(95 \% \mathrm{CI}=0.88-$ $51.31), p \leq 0.06)$. For hyperlipidemia, both groups had significant increases in the odds of compliance to statin medications (intervention odds ratio $=5.98$ (95\% CI=2.81, 12.76), $p<0.0001$ and control odds ratio $=3.83(95 \% \mathrm{CI}=1.83-8.01), p<0.0004)$; however, the comparison of these two groups showed that these increases were not significantly different. For compliance to hypertension medications, the intervention group showed significantly greater odds of compliance post-stroke (odds ratio=3.68 $(95 \% \mathrm{CI}=$ $1.81-7.48), p<0.0004)$. The control group showed no difference in compliance rates between these two time points. The comparison of the odds between the intervention and control groups showed that this increase was not significant (odds ratio $=2.34(95 \%$ $\mathrm{CI}=0.86-6.40), p<0.10)$.

\section{DISCUSSION}

This study demonstrated the feasibility of developing and delivering a locally tailored intervention for secondary stroke prevention. The intervention reached and promoted the implementation of secondary stroke self-management by veteran patients with an acute stroke/TIA. The program's effectiveness was mixed. There were no significant effects on patient self-efficacy or stroke-specific, health-related quality

Table 3 | Medication possession ratios pre (180 days) and post-stroke (180 days) 


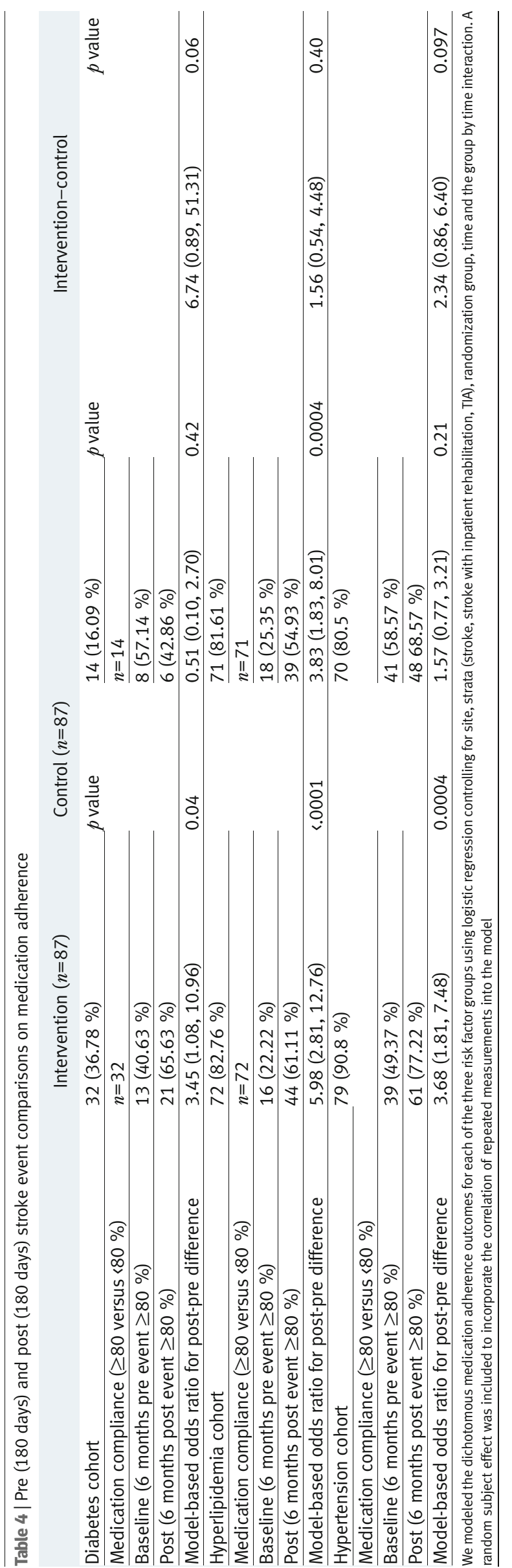


of life. However, we saw within the intervention group significant improvements in medication adherence among stroke survivors with diabetes, and hypertension. These improvements were not significantly different between the changes in the control group. Also, there was a significant decrease in diabetic medication adherence among those assigned to the control group. We also reported a significant increase in medication adherence for hypertensive stroke survivors assigned to the intervention group while adherence for those assigned to the control group remained the same. Among stroke survivors with elevated cholesterol and discharged on statin medication, there were significant increases within both groups (intervention and control). This overall increase may have reflected a statin performance measure for facilities that had been recently implemented in VHA.

These findings suggest that an acute stroke/TIA event is a fertile period to focus on medication adherence among veteran patients as guideline care calls for medication management for diabetes, hyperlipidemia, and hypertension at discharge and followup outpatient care. Our findings in increased medication adherence among stroke survivors with diabetes and hypertension are meaningful given the poor adherence rates previously reported among stroke survivors [31-33]. Furthermore, poor medication adherence by patients was identified by clinicians at both sites as a major barrier to secondary stroke prevention during our formative evaluation [7]. Moreover, our stroke self-management intervention provided an opportunity for stroke survivors to address specific medication concerns which has been previously identified as a major barrier to medication adherence in survivors of strokes and transient ischemic attacks (TIA) [35-37].

The local adaptation of the self-management portion of the intervention permitted the sites to deliver the program based on staffing and opportunities within the facilities to deliver to the stroke survivors during the acute hospitalization and the following outpatient period. Both sites utilized nurse case managers to deliver their respective programs concurrent to usual neurology care, and found comparable adherence rates in this trial as those rates reported in trials where clinical pharmacists delivered the programs. [38] The majority of participants assigned to the intervention received nurse-led support sessions. The difference between sites on the proportion of the intervention program delivery may be due in part to the larger volume (i.e., twice the rate) of acute stroke patients at site 2 compared to site 1 . Regardless of time spent with a nurse case manager, all participants randomized to the intervention group received the secondary stroke prevention materials.

While we report significant improvements in medication adherence among stroke survivors participating in the intervention with comorbid diabetes and hypertension, there were several limitations to this study. First, this was a bundled multi-level intervention, making it difficult to ascertain the effect of each component on the outcomes. However, both sites' stroke self-management programs targeted medication adherence, a common component of selfmanagement programs. Second, because we included participants with a wide range of stroke severity (both acute stroke and TIA patients) at baseline, we controlled for stroke severity at baseline by randomizing stroke subjects within strata defined by inpatient rehabilitation. Third, our sample was comprised of veteran patients who were predominantly male. Thus, our data may have limited generalizability. Fourth, both VA facilities were involved in ongoing stroke quality improvement efforts outside of this study which may have influenced clinician activities across patient groups. Finally, this evaluation includes data based upon patient medication refills. It is possible that medication may have been refilled outside the Veteran Healthcare Administration or that the refilled medication may not have been consumed as prescribed. However, veteran patients typically refill their medications within VHA, given the major financial draw of the VHA prescription benefit. Nonetheless, our pilot implementation program which permitted two large VA medical centers to locally adapt secondary stroke prevention demonstrates the potential for improving medication adherence after stroke to reduce secondary stroke risk. Prescribed medications are often the therapeutic cornerstone for secondary stroke risk factor reduction [29]. Despite some improvements in medication adherence, overall stroke-specific, health-related quality of life did not improve suggesting that other components may be necessary to promote in a stroke self-management program. We speculate that a greater focus on social and role functioning may potentially impact more global effectiveness outcomes.

Conclusions-A secondary stroke risk factor selfmanagement program for veterans with stroke/ TIA has the potential to improve medication adherence for veteran patients with comorbid cardiovascular conditions (diabetes and hypertension) after an acute stroke/TIA event. Usual care practices are already targeting patients with hyperlipidemia. Additional intervention components may be needed to enhance more global effectiveness outcomes of patient self-efficacy and stroke-specific, healthrelated quality of life.

Acknowledgments: This study was funded by the VA HSRD Investigator Initiated Research Grant IAB 05-297-2 and by the HSRD VA Stroke QUERI Center. The trial registration number is Current Clinical Trials NCT00355147. We thank the veteran participants and the staff at the VA Medical Centers for their time spent on this project.

\section{Compliance with ethical standards}

Conflict of interest-The authors declare that they have no competing interests. 


\section{Appendix A}

Table 5 | List of medications in VHA PBM prescribed for clinical conditions: diabetes, hypercholesterolemia, and hypertension Anti-hypertensives/statins

\begin{tabular}{ll}
\hline CV100 & Beta blockers/related \\
\hline CV150 & Alpha blockers/related \\
\hline CV250 & Calcium channel blockers \\
\hline CV300 & Antianginals \\
\hline CV350 & Antiarrhythmics \\
\hline CV400 & Antilipemic agents \\
\hline CV490 & Anti-hypertensive combinations \\
\hline CV800 & Anti-hypertensives, other \\
\hline CV806 & Ace inhibitors \\
\hline CV900 & Angiotensin II inhibitor \\
\hline Diuretics & Direct renin inhibitor \\
\hline CV700 & Cardiovascular agents, other \\
\hline CV701 & \\
\hline CV702 & Diuretics \\
\hline CV703 & Thiazides/related diuretics \\
\hline CV704 & Loop diuretics \\
\hline CV709 & Carbonic anhydrase inhibitor diuretics \\
\hline Diabetes medications & Potassium sparing/combinations diuretics \\
\hline HS500 & Diuretics, other \\
\hline HS501 & \\
\hline HS502 & Blood glucose regulation agents \\
\hline HS509 & Insulin \\
\hline
\end{tabular}




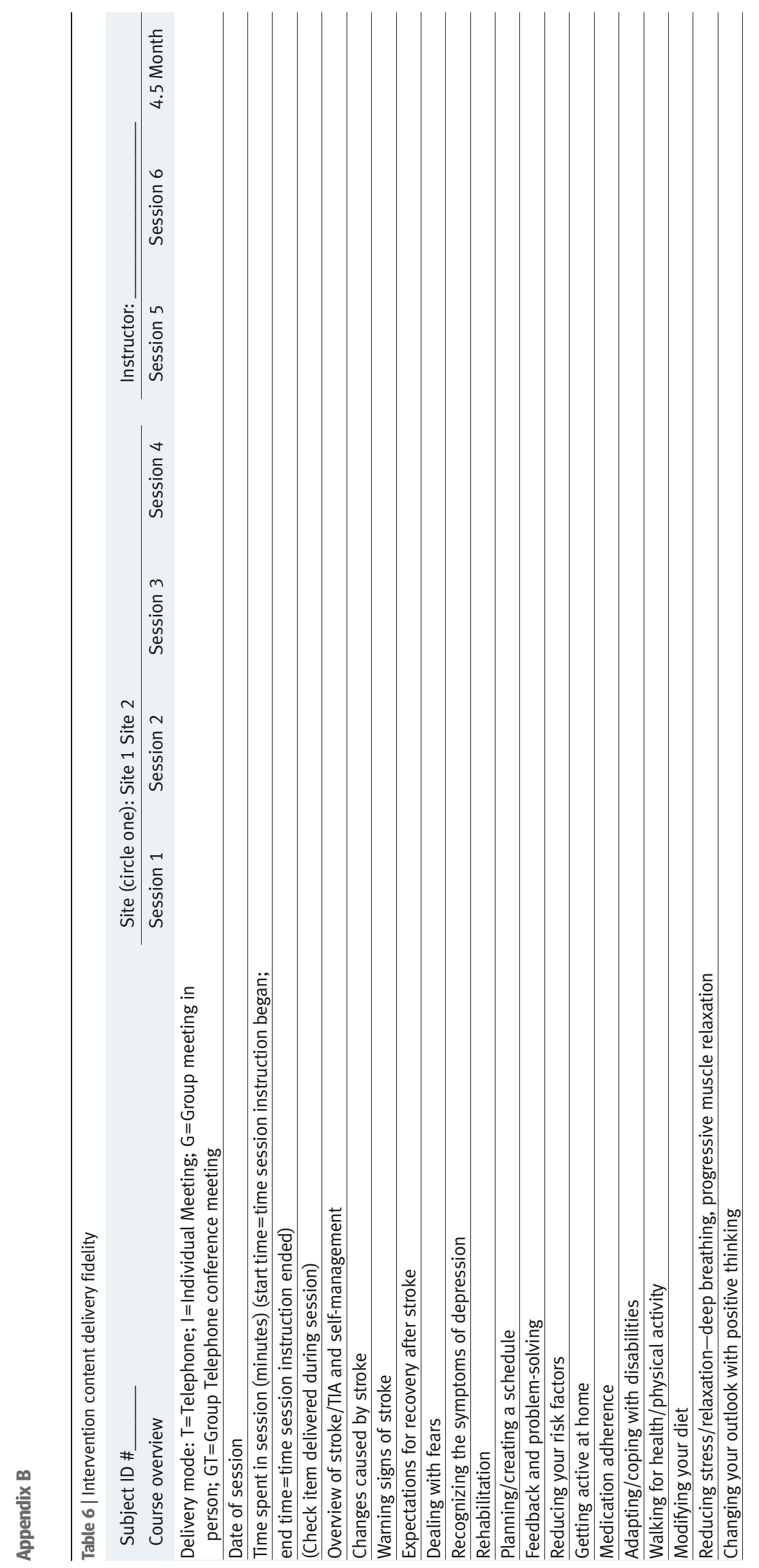


Table 7 | Common reported patient goals for stroke self-management

GOALS categories

Category number Category description

4

5

6

7

8

9

10

11

12

Communicating needs/questions with health care provider

2. Attending rehabilitation therapy appointments (OT, PT and Speech)

Doing therapeutic exercises at home

House/yard chores, dog walking, walking with or without a pedometer

Stress management activities

Improving nutrition, decreasing fat and salt,

Smoking reduction or cessation behaviors

Blood pressure management and measurement

13 Engagement in social activities or hobbies

14 Medication management /compliance

15 Other-talking with social services or other service regarding financial issues/job loss, etc.; starting to drive again

\section{References}

1. American Heart Association Statistical Update. Heart disease and stroke statistics-2014 update. A report from the American Heart Association. Circulation. 2014;129:e28-292. doi:10.1161/01.cir

2. Go A, Mozaffarian D, Roger V, et al. Heart disease and stroke statistics-2013 update. A report from the American Heart Association. Circulation. 2013; 127: e6-e245.

3. Williams LS, Harris LE, Biller J. Measuring quality of life in a way that is meaningful to stroke patients. Neurology. 1999; 53: 1839-1843.

4. Lai SM, Duncan PW, Perera S. Persisting consequences of stroke measured by the stroke impact scale. Stroke. 2002; 33: 1840-1844.

5. Damush TM, Ofner S, Yu Z, et al. Implementation of a stroke selfmanagement program: a randomized controlled pilot study of veterans with stroke. Trans Behav Med. 2011; 1(4): 561-572.

6. Wolfe CDA, Redfern J, Rudd AG, et al. Cluster randomized controlled trial of a patient and general practitioner intervention to improve the management of multiple risk factors after stroke: stop stroke. Stroke. 2010; 41: 2470-2476.

7. Schmid AA, Anderson J, Kent T, et al. Using intervention mapping to develop and adapt a secondary stroke prevention program in Veterans Health Administration Medical Centers. Implement Sci. 2010; 5: 97.

8. Veterans Health Administration DoD. VA/DoD clinical practice guideline for the management of stroke rehabilitation in the primary care setting. Washington: Department of Veteran Affairs; 2003.

9. Veterans Health Administration DoD Management of Stroke Rehabilitation Working Group. VA/DoD clinical practice guideline for the management of stroke. Washington, DC: Department of Veteran Affairs. J Rehabil Res Dev. 2010; 47(9): 1-43.

10. Fonarow GC, Reeves MJ, Wmith EE, et al. Characteristics, performance measures, and in-hospital outcomes of the first one million stroke and transient ischemic attack admissions in get with the guidelines stroke. Circ Cardiovasc Qual Outcomes. 2010; 3: 291-302.

11. Smaha LA. The American Heart Association get with the guidelines program. Am Heart J. 2004; 148(5 Suppl): S46-S48.

12. Joint Commission on Accreditation of Healthcare Organizations. ICAHO 2005; Available at: URL: www.jcaho.org.

13. Joint Commission. The Joint Commission launches new comprehensive stroke certification program. It Comm Perspect. 2012; 32(10): 6-7.

14. Bartholomew LK, Parcel GS, Kok G, et al. Planning health promotion programs: an intervention mapping approach. 2nd ed. San Francisco: Wiley; 2006.
15. Ovbiagele B, Saver JL, Fredieu A, et al. PROTECT: a coordinated stroke treatment program to prevent recurrent thromboembolic events. Neurology. 2004; 63(7): 1217-1222.

16. Coleman K, Austin BT, Brach C, et al. Evidence on the chronic care model in the new millennium. Health Aff. 2009; 28(1): 75-85.

17. Schmid AA, Damush TM, Plue L, et al. Current blood pressure selfmanagement: a qualitative study. Rehabil Nurs. 2009; 34(6): 223 229.

18. Damush TM, Plue L, Bakas T, et al. Barriers and facilitators to exercise among stroke survivors. Rehabil Nurs. 2007; 32(6): 253 260. 262.

19. Shokar NK. Translating research into practice: the colorectal cancer screening experience. J Gen Intern Med. 2013; 29(1): 10-12.

20. Williams LS, Kroenke K, Bakas T, et al. Case-management of post stroke depression: a randomized, controlled trial. Stroke. 2007; 38(3): 998-1003.

21. Callahan CM, Unverzagt FW, Hui SL, et al. Six-item screener to identify cognitive impairment among potential subjects for clinical research. Med Care. 2002; 40(9): 771-781.

22. Welch DC, West RL. The short portable mental status questionnaire: assessing cognitive ability in nursing home residents. Nurse Res. 1999; 48(6): 329-332.

23. Lyden P, Raman R, Liu L, et al. National Institutes of Health Stroke scale. Certification is reliable across multiple venues. Stroke. 2009; 40(7): 2507-2511.

24. Bandura A. Social foundations of thoughts and action: a social cognitive theory. Englewood Cliffs: Prentice-Hall; 1986.

25. Lorig KR, Ritter P, Stewart AL, et al. Chronic disease selfmanagement program: 2 year health status and health care utilization outcomes. Med Care. 2001; 39: 1217-1223.

26. Lorig KR. Self-management education: more than a nice extra. Med Care. 2003; 41: 699-701.

27. Gaglio B, Glasgow RE. Evaluation approaches for dissemination and implementation research. In: Brownson R, Colditz G, Proctor E, eds. Dissemination and implementation research in health. 1st ed. New York: Oxford University Press; 2012: 327-356.

28. Lorig K, Stewart AL, Ritter P, et al. Outcome measures for health education and other health care interventions. Thousand Oaks: Sage Publications; 1996.

29. Go AS, Mozaffarian D, Roger VL, et al. Heart disease and stroke statistics-2014 update a report from the American Heart Association Writing Group Members. Circulation. 2014; 129: e28-e292.

30. Zillich AJ, Jaynes HAW, Snyder ME, et al. Evaluation of specialized medication packaging combined with medication therapy

page 467 of 468 
management: adherence, outcomes, and costs among Medicaid patients. Med Care. 2012; 50: 485-493.

31. Steiner JF, Prochazka AV. The assessment of refill compliance using pharmacy records: methods, validity, and applications. J Clin Epidemiol. 1997; 50: 105-116.

32. Peterson AM, Nau DP, Cramer JA, et al. A checklist for medication compliance and persistence studies using retrospective databases. Value Health. 2007; 10: 3-12.

33. Bushnell CD, Zimmer LO, Pan W, et al. Persistence with stroke prevention medications 3 months after hospitalization. Arch Neurol. 2010; 67: 1456-1463.

34. Williams L, Weinberger M, Harris L, et al. Development of a strokespecific quality of life (SS-QOL) scale. Stroke. 1999; 30: 1362-1369.
35. Khan NA, Yun L, Humphries K, et al. Antihypertensive drug use and adherence after stroke: are there sex differences? Stroke. 2010; 41: 1445-1449.

36. Kronish IM, Diefenbach MA, Edmondson DE, et al. Key barriers to medication adherence in survivors of strokes and transcient ischemic attacks. J Gen Int Med. 2013; 28(5): 675-682.

37. O'Carroll R, Whittaker J, Hamilton B, et al. Predictors of adherence to secondary preventive medication in stroke patients. Ann Behav Med. 2011; 41: 383-390.

38. Hohmann C, Neumann-Haefelin T, Klotz JM, et al. Adherence to hospital discharge medication in patients with ischemic stroke: a prospective, interventional 2-phase study. Stroke. 2013; 44(2): 522-524. 